\title{
A Novel First-Line Treatment Alternative for Noncomplicated İdiopathic Granulomatous Mastitis: Combined Intralesional Steroid İnjection with Topical Steroid Administration
}

\author{
Osman Toktas $^{a}$ Can Konca $^{b}$ Didem Can Trabulus ${ }^{c}$ Aykut Soyder ${ }^{d}$ \\ Hande Koksal $^{\mathrm{e}}$ Hasan Karanlik ${ }^{\mathrm{f}}$ Ayfer Kamali Polat ${ }^{\mathrm{g}}$ Serdar Ozbas $^{\mathrm{h}}$ \\ Serdar Yormaz ${ }^{i}$ Arda Isik $^{j}$ Efe Sezgink Atilla Soran'

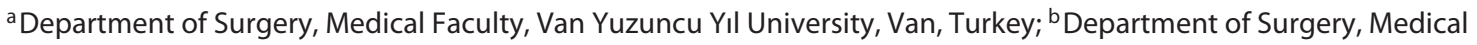 \\ Faculty, Ankara University, Ankara, Turkey; ${ }^{c}$ Department of Surgery, Istanbul Samatya Training and Research

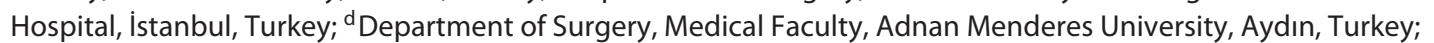 \\ e Department of Surgery, Konya Training and Research Hospital, Konya, Turkey; ${ }^{\mathrm{f}}$ Department of Surgery, Istanbul \\ University Institute of Oncology, İstanbul, Turkey; ${ }^{9}$ Department of Surgery, Medical Faculty, 19 Mayıs University,

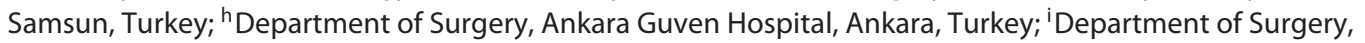 \\ Medical Faculty, Selcuk University, Konya, Turkey; jDepartment of Surgery, Medical Faculty, Erzincan Binali Yıldırım \\ University, Erzincan, Turkey; ${ }^{\text {}}$ Izmir Instıtute of Technology, Izmir, Turkey; 'Department of Surgery, University of \\ Pittsburgh, Pittsburgh, PA, USA
}

\section{Keywords \\ Idiopathic granulomatous mastitis (IGM) - Non-complicated IGM · Steroid injection · Topical steroid · Systemic steroid}

\section{Abstract}

Background: Idiopathic granulomatous mastitis (IGM) is a rare form of nonlactational mastitis. Due to the small number of case series and consequently inadequate prospective studies, there is still no consensus on the optimal treatment of IGM. In this study, we aimed to compare the efficacy of intralesional steroid injection with concomitant topical steroids to systemic steroid therapy only in the treatment of noncomplicated IGM. Methods: Between June 2015 and April 2018, the patients' data was prospectively collected and analyzed retrospectively. The study included a total of 78 female patients diagnosed with IGM. Patients were divided into 2 groups: the local steroid treatment group (intralesional steroid injection with topical steroid administration; group $1, n=46$ ) and the peroral systemic steroid treatment group (group 2, $n=32$ ). Response to the therapy, side effects, recurrence, the need for surgical treatment, and complication rates were compared. Results: Forty-three patients
\end{abstract}

(93.5\%) in group 1 achieved a partial or complete response compared to 23 patients (71.9\%) in group 2 after 3 months; this difference was significant $(p=0.012)$. The recurrence rates were significantly lower in group $1(8.7 \%)$ compared to group 2 (46.9\%; $p=0.001)$, and the need for surgical treatment was significantly less in group $1(2.2 \%)$ than in group 2 (9.4\%; $p=0.001)$. While the complication rates were similar between groups, a higher rate of systemic side effects was observed in group 2. Conclusion: Based on the results of our study, combined steroid injection and topical steroid treatment in IGM is as effective as systemic steroid treatment. We suggest that this combination therapy of topical steroids and local steroid injection should be used as first-line therapy in patients with noncomplicated IGM.

(c) 2020 S. Karger AG, Basel

\section{Introduction}

Idiopathic granulomatous mastitis (IGM) is a rare form of nonlactational mastitis [1]. Initially described by Kessler and Wolloch [2], this inflammatory and be- 
nign condition is still a challenging disease to treat. There are many different mechanisms suggested in the literature on how IGM develops, yet its etiology is still unknown [1]. Autoimmune response, infection, and hormonal disruption are considered the 3 leading hypotheses, though autoimmune response is the most accepted theory [3]. Clinically associated with hyperprolactinemia, IGM is usually observed as a persistent or recurrent tender mass in the breast in parous premenopausal women with a history of lactation [1]. Since the clinical presentation of IGM is similar to that of breast cancer and infectious or inflammatory breast diseases, histopathological examination is required for definitive diagnosis [1]. Core-needle biopsy is the preferred method, with a diagnostic accuracy of $94.5 \%$ [4], though incisional and excisional biopsies are alternative diagnostic methods [1]. Histopathologically, IGM is characterized by noncaseating granulomas around lobules and ducts in the breast. Specific causes of infection, trauma, and foreign body reactions should also be excluded [5]. Due to the small number of case series and lack of prospective studies, there is still no consensus on the optimal treatment. While wide surgical excisions have been used in the treatment in the 1980s, conservative approaches are preferred and current best practice avoids surgical management unless absolutely necessary. Some authors advocate expectant management of the disease with active surveillance after antibiotic treatment. However, this process may take months or even years in some patients [6,7]. Although most patients receive antibiotic treatment before the definitive diagnosis because of diagnostic uncertainty, routine antibiotic use is not recommended as IGM is a sterile condition [8]. Given the leading hypothesis of an autoimmune etiology, there are many studies in the literature documenting therapeutic efficacy of immunosuppressant agents such as corticosteroids, methotrexate, azathioprine, bromocriptine, and colchicine [9]. Because of high recurrence rates, delayed wound healing, and poor cosmetic results, surgical interventions are generally employed only for aggressive disease or in cases where there is no response to medical treatment $[1,10]$.

Corticosteroids, first used by DeHertogh et al. [11], have become the most commonly used agents today. The efficacy of systemic corticosteroid therapy has been the subject of many studies to date and has been shown to provide a faster recovery than expectant management. However, systemic steroids have a wide range of side effects that can limit their use $[12,13]$. The use of local corticosteroids is generally better tolerated, and there are several studies that document the efficacy of topical corticosteroid therapy in the treatment of IGM $[13,14]$. The results of these studies show that topical treatment is also effective, but recovery takes a longer time compared to systemic treatment. However, there is no data in the literature on intralesional steroid injection, which is the subject of our study.

In this study, we aimed to compare the efficacy of combined intralesional steroid injection with topical steroid administration to systemic steroid therapy in the treatment of noncomplicated IGM.

\section{Materials and Methods}

This multicenter prospectively maintained registry study protocol was established by the Turkish Breast Disease Working Group (TBDWG). The data collected between June 2015 and April 2018 was analyzed retrospectively. Patients who presented to the breast centers of this study with complaints of breast mass, breast pain, and local erythema were evaluated clinically and radiologically. Patients with suspected IGM underwent core needle, incisional, or excisional biopsy according to their clinical presentation and local protocol of the facility. The diagnosis of IGM was made histopathologically by pathologists of each center. In the histopathological diagnosis of each patient, infectious granulomatous mastitis agents were excluded with Gram, periodic acid-Schiff, Ziehl-Neelsen, and Giemsa stains. Patients who were diagnosed with IGM and received peroral or local administration of corticosteroids with at least 12 months of follow-up were included in the study. Patients who clinically or radiologically had a breast abscess or fistula, patients who received a previous medical treatment for IGM, those with recurrent disease or a contraindication for the use of corticosteroids, and those who were pregnant or lactating were excluded from the study. Patients' age, comorbid medical conditions, number of pregnancies and live births, oral contraceptive use, smoking, complaint at disease onset, radiologic method preferred for the diagnosis and follow-up (such as breast ultrasound, mammography, and breast MRI, depending on the patients age and clinical status), and the biopsy method used for diagnosis were abstracted from the patients' records.

\section{Treatment Protocol}

Patients were divided into 2 groups: the local steroid treatment group (group 1, $n=46$ ) and the peroral steroid treatment group (group 2, $n=32$ ). The local steroid treatment protocol included an intralesional triamcinolone acetonide (Kenacort-A amp. $40 \mathrm{mg}$ / $\mathrm{mL}$; Deva, Istanbul, Turkey) injection $20 \mathrm{mg} / 1 \mathrm{~cm}^{3}$ in the center of lesions once a month under breast ultrasound guidance (Fig. 1) and topical administration of triamcinolone acetonide $0.1 \%$ pomade (Kenacort-A \% 0.1, 20-g pomade; Deva) on the skin of the affected region of the breast twice a day on every other day for 1 month. Patients with diffuse disease were injected at different points with an interval of $5 \mathrm{~cm}$. In the patients with multiple lesions, if the lesions were $>5 \mathrm{~cm}$ away from each other, lesions were injected separately; if the lesions were $<5 \mathrm{~cm}$ from each other, the larger one was injected. Oral steroid treatment was administered as methylprednisolone $32 \mathrm{mg} /$ day for a month. The treatment protocol was administered based on the clinical experience of the physician and the treatment center. After the treatment options were explained to the patient, a decision was made mutually by the physician and the patient. Per protocol, patients with abscess and fistula were excluded from the study.

At the end of the first month, all patients were evaluated clinically and radiologically for treatment response, which was categorized as "complete response" for the patients with complete reso- 


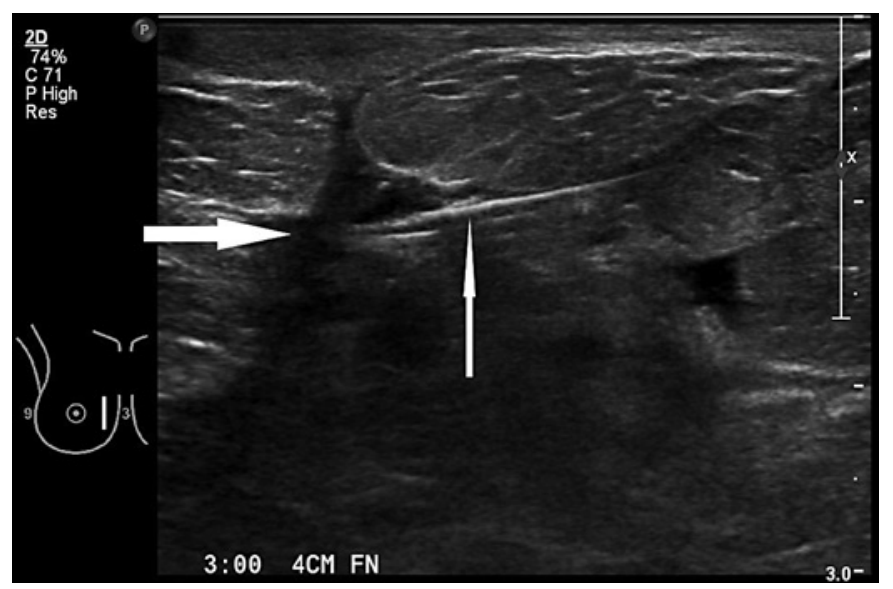

Fig. 1. Ultrasound-guided steroid injection (thin arrow is the needle in the center of the IGM lesion; thick arrow is the center of the IGM lesion, right breast).

lution of the disease clinically and radiologically, "partial response" for the patients with partial resolution of the disease, "no response" for the patients without improvement in symptoms and radiological findings, and "worsening disease" for the patients with worsening of clinical symptoms and radiological findings. Patients with "complete response" were enlisted to follow-up (Fig. 2), while patients with "partial response," "no response," and "worsening disease" received a second and, if necessary, a third course of treatment with monthly check-ups until the resolution of the disease. All patients were informed about the course of the disease, available treatment options, and their rate of success at the initial and follow-up examinations. Surgical treatment was performed for patients who clinically required or preferred surgery during follow-up.

\section{Statistical Analysis}

Continuous and categorical variable differences between the 2 groups were analyzed by $t$ tests and $\chi^{2}$ tests, respectively. $p$ values $<0.05$ were considered as statistically significant. All analyses were conducted with R program version 3.2.3 (R Foundation for Statistical Computing, Vienna, Austria; https://www.r-project.org/).

\section{Results}

The study included a total of 78 female patients diagnosed with IGM. There were 46 patients who received local steroid treatment (group 1) and 32 patients who received systemic steroid treatment (group 2). The mean age was similar in both groups $(p=0.94)$. Comorbid medical conditions such as diabetes mellitus, hypertension, and cardiac disease were found in $6(13.1 \%)$ of the patients in group 1 while there were no comorbidities in the patients of group $2(p=0.01)$. The majority of the patients diagnosed with IGM had at least 1 child $(84.8 \%$ in group 1 and $68.8 \%$ in group 2 ); the number of patients with no parity was $3(6.5 \%)$ in group 1 and there were no such patients in group 2 . Total breastfeeding time of the patients

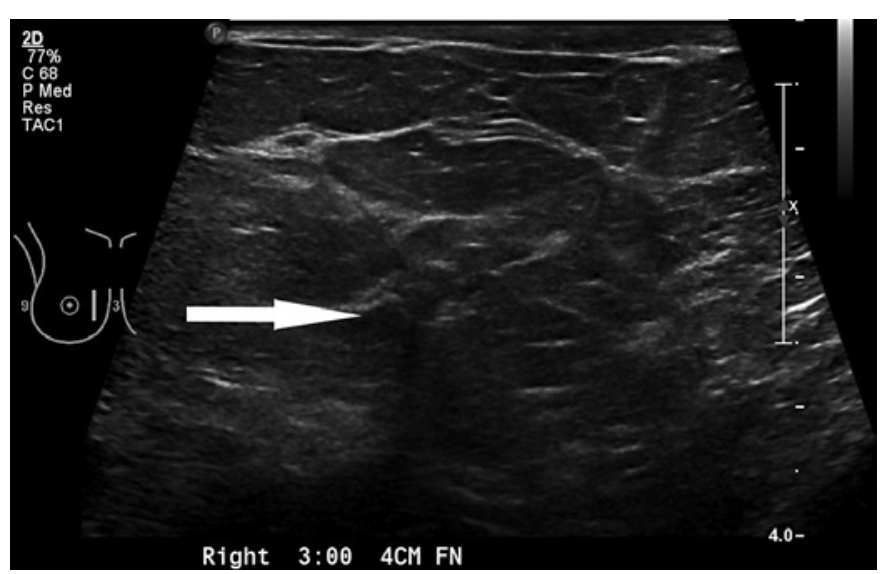

Fig. 2. Two-month follow-up (arrow; the center of the IGM lesion near-complete response, right breast).

Table 1. Patients and their clinical characteristics

\begin{tabular}{|c|c|c|}
\hline & $\begin{array}{l}\text { Group 1 } \\
(n=46)\end{array}$ & $\begin{array}{l}\text { Group } 2 \\
(n=32)\end{array}$ \\
\hline Age, years & $36.5 \pm 1.1$ & $36.7 \pm 1.4$ \\
\hline \multicolumn{3}{|l|}{ Comorbid medical conditions } \\
\hline One or more & $6(13.9)$ & $0(0)$ \\
\hline None & $40(86.1)$ & $32(100)$ \\
\hline \multicolumn{3}{|l|}{ Parity } \\
\hline None & $3(6.5)$ & $0(0)$ \\
\hline 1 & $18(39.1)$ & $4(12.5)$ \\
\hline 2 & $16(34.8)$ & $12(37.5)$ \\
\hline 3 or more & $9(19.6)$ & $16(50.0)$ \\
\hline \multicolumn{3}{|c|}{ Total breastfeeding time, months } \\
\hline & $16.4 \pm 2.3$ & $32.2 \pm 6.8$ \\
\hline \multicolumn{3}{|l|}{ Smoking } \\
\hline Smoker & $10(21.7)$ & $6(18.8)$ \\
\hline Nonsmoker & $36(78.3)$ & $26(82.2)$ \\
\hline \multicolumn{3}{|l|}{ Oral contraceptive use } \\
\hline Positive & $11(23.9)$ & $0(0)$ \\
\hline Negative & $35(76.1)$ & $32(100)$ \\
\hline \multicolumn{3}{|l|}{ Localization of disease } \\
\hline Unilateral & $44(95.6)$ & $31(96.9)$ \\
\hline Bilateral & $2(4.4)$ & $1(3.1)$ \\
\hline \multicolumn{3}{|l|}{ Main complaint at onset } \\
\hline Mass + pain & $20(43.4)$ & $7(27.8)$ \\
\hline Mass + pain + inflammation & $26(56.6)$ & $25(72.2)$ \\
\hline \multicolumn{3}{|c|}{ Preferred radiological examination method } \\
\hline USG & $16(34.8)$ & $30(93.8)$ \\
\hline$U S G+M M G$ & $13(28.3)$ & $2(6.2)$ \\
\hline$U S G+M M G+M R I$ & $17(36.9)$ & $0(0)$ \\
\hline \multicolumn{3}{|c|}{ Preferred tissue sampling method for diagnosis } \\
\hline Core needle & $45(97.8)$ & $24(75.0)$ \\
\hline Incisional & $1(2.2)$ & $7(21.9)$ \\
\hline Excisional & $0(0)$ & $1(3.1)$ \\
\hline
\end{tabular}

Values represent mean $\pm \mathrm{SD}$ or $n(\%)$, as appropriate. 
Table 2. Response rates of patients in treatment arms

\begin{tabular}{|c|c|c|c|c|c|c|}
\hline & \multicolumn{2}{|c|}{ 1st administration } & \multicolumn{2}{|c|}{ 2nd administration } & \multicolumn{2}{|c|}{ 3rd administration } \\
\hline & $\begin{array}{l}\text { group } 1 \\
(n=46)\end{array}$ & $\begin{array}{l}\text { group } 2 \\
(n=32)\end{array}$ & $\begin{array}{l}\text { group } 1 \\
(n=32)\end{array}$ & $\begin{array}{l}\text { group } 2 \\
(n=26)\end{array}$ & $\begin{array}{l}\text { group } 1 \\
(n=8)\end{array}$ & $\begin{array}{l}\text { group } 2 \\
(n=12)\end{array}$ \\
\hline Complete response & $8(17.4)$ & 7 (21.9) & $21(65.6)$ & $18(69.2)$ & $5(62.5)$ & $9(75.0)$ \\
\hline Partial response & $35(76.1)$ & $16(50.0)$ & $9(28.1)$ & $8(30.8)$ & $3(37.5)$ & $2(16.7)$ \\
\hline No response & $2(4.4)$ & $9(28.1)$ & $2(6.3)$ & $0(0)$ & $0(0)$ & $1(8.3)$ \\
\hline Worsening disease & $1(2.2)$ & $0(0)$ & $0(0)$ & $0(0)$ & $0(0)$ & $0(0)$ \\
\hline
\end{tabular}

Values represent $n(\%)$.

Table 3. Clinical follow-up and treatment results of the groups

\begin{tabular}{lrcl}
\hline & $\begin{array}{c}\text { Group 1 } \\
(n=46)\end{array}$ & $\begin{array}{l}\text { Group 2 } \\
(n=32)\end{array}$ & $\begin{array}{l}p \\
\text { value }\end{array}$ \\
\hline Follow-up time, months & $17.5 \pm 3.7$ & $23.2 \pm 9.1$ & 0.005 \\
Recurrence & $4(8.7)$ & $15(46.9)$ & 0.001 \\
Surgery & $1(2.2)$ & $4(12.5)$ & 0.001 \\
Complication & $1(2.2)$ & $3(9.4)$ & 0.16 \\
\hline
\end{tabular}

Values represent mean $\pm \mathrm{SD}$ or $n(\%)$, as appropriate.

was less in group $1(p<0.0001)$. Smoking ratio was similar between the 2 groups $(p=0.75)$. While $11(23.9 \%)$ patients were on oral contraceptives in group 1 , there were no patients using oral contraceptives in group $2(p=$ $0.0003)$. None of the patients in the treatment arms were taking immunosuppressive treatment at the time of diagnosis. While the main complaint at onset was predominantly a breast mass or local pain and inflammation in group 1, the majority of group 2 patients presented with all 3 signs of disease as a breast mass with pain and signs of local inflammation. Patients and their clinical characteristics are shown in Table 1.

In group 1, $6(15.8 \%)$ out of 38 patients who did not achieve a complete response after the first course of treatment and $3(27.3 \%)$ out of 11 after the second course of treatment were lost to follow-up. In group 2, 1 patient with a complete response after the first course of treatment received a second course of treatment due to recurrence. Similarly, a third course of treatment was given to 4 patients in group 2 who developed recurrence after the second course of treatment (Table 2).

When patients with complete and partial response were pooled in a single group to determine the rate of "responders," 43 patients (93.5\%) were responders in group 1 and 23 (71.9\%) in group 2 (Table 2). Accordingly, treatment response rate was significantly higher in group 1 than in group $2(p=0.012)$. The recurrence rates were significantly lower in group $1(8.7 \%)$ compared to group $2(46.9 \% ; p=0.001)$, and the need for surgical treatment was almost 4 times less in group $1(2.2 \%)$ than in group 2 $(12.5 \% ; p=0.001$; Table 3$)$.

Surgery was performed on patients who developed abscess or fistula, those without response after the second treatment, and those with recurrent disease. In group 1, 1 (2.2\%) patient without response requested surgical management and total mastectomy was performed because of diffuse mastitis. In group 2, 4 (12.5\%) patients who had either no response or recurrent disease were treated with surgery (Table 3); 1 of the patients underwent total mastectomy as she had diffuse mastitis and 3 underwent lumpectomies.

Complication rates were similar between groups $(p=$ $0.16)$. Thinning of the skin as a topical side effect of corticosteroids was observed in 1 patient in group 1. By comparison, 3 patients in group 2 had systemic side effects such as hirsutism in 2 patients and weight gain in 1 patient (Table 3).

\section{Discussion}

IGM is a rare chronic benign disease of the breast. Generally observed in breastfeeding women, the suspected etiologies of this condition include autoimmunity to proteins secreted to ducts, undetectable microorganisms, or breastfeeding reactions $[14,15]$. There is still no consensus on the optimal treatment modality, the type and timing of the drug used, and the indication/extent of surgery. Today, surgery is avoided as much as possible and immunosuppressive agents, predominantly corticosteroids, are preferred in the treatment of IGM. However, the systemic immunosuppressive agents come with a drawback of several side effects $[7,16,17]$. In this study, we aimed to compare the efficacy of combined intralesional steroid injection with topical steroid administration, which may have a better side effect profile to systemic corticosteroid treatment. 
In our study, we found that local steroid treatment is as effective as systemic treatment. In the literature, systemic steroid treatment, surgery, or active surveillance are among the frequently reported modalities of treatment for IGM. There are conflicting results when systemic steroid treatment is compared with surgical treatment or active surveillance. However, given the poor cosmetic results, delayed wound healing, and high recurrence rates seen with surgical treatment, systemic corticosteroid use has been the mainstay of medical treatment in IGM, although no consensus has yet been reached [13, $14,18,19]$. Today, systemic steroid therapy is used in long-term control of the disease, especially in patients with recurrence. However, side effects associated with long-term use such as weight gain, hirsutism, diabetes, and Cushing's syndrome lead to limitations in treatment [20]. Local administration of corticosteroids has the advantage of a better side effect profile compared to systemic treatment; however, the data on the use of local steroid treatment is limited. There are only a few studies investigating the use of topical steroid application in the treatment of IGM. In a recent study by Çetin et al. [13] comparing topical and systemic steroid treatment in IGM, the efficacy of topical treatment was similar to that of systemic treatment. However, subjects responded faster to systemic treatment, with a mean recovery period of 11.7 weeks on systemic therapy compared to 22 weeks with topical treatment. In the same study, the local side effects of steroids were $24.4 \%$ and the systemic side effects were $2.4 \%$ in patients receiving topical treatment, whereas systemic side effects were observed in $38.2 \%$ of patients receiving systemic steroids. In a study by $\mathrm{Al}-$ tintoprak et al. [14] where IGM was treated with topical steroids, clinical improvement occurred in an average of 8.3 weeks in 28 IGM patients with a long-term success rate of over $90 \%$ during a mean follow-up of 37.2 months. There were no reported topical steroid-related side effects during the treatment period. While there are studies investigating the use of topical steroids in IGM, there are no references in the literature other than case reports on the use of local corticosteroid injection. In this respect, our study is the first to compare the treatment efficacy of local corticosteroid injection with its systemic use. When the results of our study are examined in terms of response to treatment, it is observed that local corticosteroid injection in combination with topical corticosteroid administration is as effective as systemic corticosteroid treatment. Furthermore, when the patients who received complete and partial response were pooled in a single group, it was observed that the response rate was $93.5 \%$ in the steroid-injected group and $71.9 \%$ in the systemic steroid group. Considering this statistically significant difference, local steroid injection may even be more effective than systemic treatment.

A Novel First-Line Treatment Alternative for Noncomplicated IGM
In our study, the locally treated group had lower rates of steroid-associated side effects. Local side effects were observed in $2.2 \%$ of patients receiving topical treatment with steroid injections while systemic side effects were observed in $9.4 \%$ of patients receiving systemic treatment. The rate of systemic side effects in the peroral steroid treatment arm is also lower than those reported in the literature. The low rate of systemic side effects observed in our study could be attributed to the relatively shorter exposure to systemic steroids with a maximum duration of treatment of 3 months.

The response rates observed in our study suggest that the efficacy of steroid treatment in both groups becomes more prominent in the second month of treatment while the rate of complete response reaches $89.5 \%$ (34 out of 38 patients) at the end of the third month. It should be noted that 9 out of 10 patients in both groups received complete response to treatment at the end of 3 months. Therefore, we recommend that treatment should be performed for at least 3 months in order to achieve the optimal efficacy of local treatment in patients who do not respond completely. On the other hand, the necessity of surgical or additional treatments should be considered in patients with worsening disease or complications.

The reported recurrence rates of IGM in the current literature range from 5.5 to $50 \%$ in a follow-up time ranging from 1.26 to 118.8 months [10, 11, 15, 21, 22]. In a retrospective study of 720 patients by Uysal et al. [9], recurrence was found in $122(17 \%)$ patients and it was found that the treatment modality had no effect on recurrence. In a review by Kuba et al. [23], recurrence rates were reported to be $23.6 \%$ after surgery and $25.8 \%$ after steroid treatment in 248 patients. In our study, the recurrence rate after steroid injection was $8.7 \%$, which is at the lower end of the spectrum compared with the reported recurrence rates in the literature and significantly lower than the rate of recurrence after systemic steroid treatment, which was $46.9 \%$ in our study. Thus, we suggest that steroid injection treatment provides better disease control than systemic treatment. That result could be explained by the higher bioavailability and dose administered per unit area with local administration of steroids; however, to confirm that hypothesis, further experimental and prospective studies are needed.

In our study, high partial response rates were reached even after the first month of steroid injection. Since that is a faster time to response than the reported times in the literature with local steroid treatments, we believe that the delay in topical treatment response is prevented by the addition of steroid injection in our study. Thus, patients with IGM might benefit from steroid injection treatments as an add-on to the conventional medical treatment chosen and this might contribute to the acceleration of treat- 
ment response. Local steroid injections could also be paired with surgery in the treatment of patients who do not respond completely and might allow the use of a less aggressive surgery.

Limitations of this study include (i) a nonrandomized design that might result in treatment allocation bias, (ii) short patient follow-up times, which might have an impact on the recurrence rates, and (iii) the limited number of patients included with some variances in baseline characteristics. Due to the study design, our study cannot comment on the relative benefit/harm between steroid injection and topical steroid treatment as they were used in combination. Prospective randomized trials with a higher number of patients and alternative protocols are required for a more comprehensive understanding of the efficacy and treatment-related side effects of local steroid injections in noncomplicated IGM.

\section{Conclusion}

Steroid injection is a treatment with a comparable efficacy, short response time, and low recurrence rate that is easy to administer and minimizes the risk of systemic side effects. We believe that even in clinically severe cases, it might be used in combination with other therapies to improve treatment efficacy and create a transition for less aggressive treatments. Prospective randomized studies investigating the effect of different doses of local steroid injections, long-term responses, and side effects should be carried out to aid the development of future treatment algorithms for patients with IGM.

\section{Acknowledgments}

The authors sincerely thank everyone who contributed from the beginning of the article to its publication.

\section{Statement of Ethics}

This study was approved by the local Ethics Committee of Van Yüzüncü Yıl University, Turkey (Reg. No. 2019/11-06). Informed consent was not received duo to the retrospective registry nature of the study.

\section{Disclosure Statement}

The authors declare that there are no potential conflicts of interest with respect to the research, authorship, and/or publication of this article.

\section{Funding Sources}

The authors received no financial support for the research, authorship, and/or publication of this article.

\section{Author Contributions}

O.T., C.K., D.C.T., A.S., H. Koksal, H. Karanlık, A.K.P., S.O., S.Y., A.I., E.S., and A.S. contributed to drafting the original grant proposal and obtained funding. O.T., C.K., and A.S. drafted the manuscript. O.T., E.F., and A.S. performed the analyses. All authors contributed to the interpretation of the data, and all authors revised the manuscript and approved the final version to be published.

\section{References}

1 Pluguez-Turull CW, Nanyes JE, Quintero CJ, Alizai H, Mais DD, Kist KA, et al. Idiopathic Granulomatous Mastitis: Manifestations at Multimodality Imaging and Pitfalls. Radiographics. 2018 Mar-Apr;38(2):330-56.

2 Kessler E, Wolloch Y. Granulomatous mastitis: a lesion clinically simulating carcinoma. Am J Clin Pathol. 1972 Dec;58(6):642-6.

3 Sheybani F, Naderi HR, Gharib M, Sarvghad M, Mirfeizi Z. Idiopathic granulomatous mastitis: long-discussed but yet-to-be-known. Autoimmunity. 2016 Jun;49(4):236-9.

4 Aghajanzadeh M, Hassanzadeh R, Alizadeh Sefat S, Alavi A, Hemmati H, Esmaeili Delshad MS, et al. Granulomatous mastitis: Presentations, diagnosis, treatment and outcome in 206 patients from the north of Iran. Breast. 2015 Aug;24(4):456-60.

5 Barreto DS, Sedgwick EL, Nagi CS, Benveniste AP. Granulomatous mastitis: etiology, imaging, pathology, treatment, and clinical findings. Breast Cancer Res Treat. 2018 Oct; 171(3):527-34.
6 Lai EC, Chan WC, Ma TK, Tang AP, Poon CS, Leong HT. The role of conservative treatment in idiopathic granulomatous mastitis. Breast J. 2005 Nov-Dec;11(6):454-6.

7 Prasad S, Jaiprakash P, Dave A, Pai D. Idiopathic granulomatous mastitis: an institutional experience. Turk J Surg. 2017 Jun; 33(2):100-3

8 Joseph KA, Luu X, Mor A. Granulomatous mastitis: a New York public hospital experience. Ann Surg Oncol. 2014 Dec;21(13): 4159-63.

9 Uysal E, Soran A, Sezgin E; Granulomatous Mastitis Study Group. Factors related to recurrence of idiopathic granulomatous mastitis: what do we learn from a multicentre study? ANZ J Surg. 2018 Jun;88(6):635-9.

10 Chirappapha P, Thaweepworadej P, Supsamutchai C, Biadul N, Lertsithichai P. Idiopathic granulomatous mastitis: A retrospective cohort study between 44 patients with different treatment modalities. Ann Med Surg (Lond). 2018 Nov;36:162-7.
11 DeHertogh DA, Rossof AH, Harris AA, Economou SG. Prednisone management of granulomatous mastitis. N Engl J Med. 1980 Oct; 303(14):799-800.

12 Erozgen F, Ersoy YE, Akaydin M, Memmi N, Celik AS, Celebi F, et al. Corticosteroid treatment and timing of surgery in idiopathic granulomatous mastitis confusing with breast carcinoma. Breast Cancer Res Treat. 2010 Sep;123(2):447-52.

13 Çetin K, Sıkar HE, Göret NE, Rona G, Barışık NÖ, Küçük HF, et al. Comparison of Topical, Systemic, and Combined Therapy with Steroids on Idiopathic Granulomatous Mastitis: A Prospective Randomized Study. World J Surg. 2019 Nov;43(11):2865-73.

14 Altintoprak F, Kivilcim T, Yalkin O, Uzunoglu Y, Kahyaoglu Z, Dilek ON. Topical Steroids Are Effective in the Treatment of Idiopathic Granulomatous Mastitis. World J Surg. 2015 Nov;39(11):2718-23. 
15 Co M, Cheng VC, Wei J, Wong SC, Chan SM, Shek T, et al. Idiopathic granulomatous mastitis: a 10-year study from a multicentre clinical database. Pathology. 2018 Dec;50(7):7427.

16 Deng JQ, Yu L, Yang Y, Feng XJ, Sun J, Liu J, et al. Steroids administered after vacuum-assisted biopsy in the management of idiopathic granulomatous mastitis. J Clin Pathol. 2017 Oct;70(10):827-31.

17 Mahmodlou R, Dadkhah N, Abbasi F, Nasiri J, Valizadeh R. Idiopathic granulomatous mastitis: dilemmas in diagnosis and treatment. Electron Physician. 2017 Sep;9(9): 5375-9.
18 Altintoprak F, Kivilcim T, Ozkan OV. Aetiology of idiopathic granulomatous mastitis. World J Clin Cases. 2014 Dec;2(12):852-8

19 Karanlik H, Ozgur I, Simsek S, Fathalizadeh A, Tukenmez M, Sahin D, et al. Can Steroids plus Surgery Become a First-Line Treatment of Idiopathic Granulomatous Mastitis? Breast Care (Basel). 2014 Oct;9(5):338-42.

20 Gautham I, Radford DM, Kovacs CS, Calhoun BC, Procop GW, Shepardson LB, et al. Cystic neutrophilic granulomatous mastitis: the Cleveland Clinic experience with diagnosis and management. Breast J. 2019 Jan;25(1): $80-5$.
21 Gautier N, Lalonde L, Tran-Thanh D, El Khoury M, David J, Labelle M, et al. Chronic granulomatous mastitis: Imaging, pathology and management. Eur J Radiol. 2013 Apr; 82(4):e165-75.

22 Bani-Hani KE, Yaghan RJ, Matalka II, Shatnawi NJ. Idiopathic granulomatous mastitis: time to avoid unnecessary mastectomies. Breast J. 2004 Jul-Aug;10(4):318-22.

23 Kuba S, Yamaguchi J, Ohtani H, Shimokawa I, Maeda S, Kanematsu T. Vacuum-assisted biopsy and steroid therapy for granulomatous lobular mastitis: report of three cases. Surg Today. 2009;39(8):695-9. 